\title{
Assessment of the Seismic Hysteresis Behavior of the Composite Frame Considering Bracing
}

\author{
Mobin Mohammadzadeh Rad ${ }^{1}$, Arash Sabzi ${ }^{2}$ \\ ${ }^{1}$ Graduate Student, Department of Civil Engineering and Faculty Member, Babol Noshirvani Industrial \\ University of Technology, Babol, Mazandaran, IRAN \\ mobin_n95@yahoo.com \\ ${ }^{2}$ Graduate Student, Department of Civil Engineering and Faculty Member, Babol Noshirvani Industrial \\ University of Technology, Babol, Mazandaran, IRAN \\ Arash.sabzi@gmail.com
}

\begin{abstract}
The present article investigates the nonlinear behavior of the mixed frames with steel beams. To do so, two different specimens of moment frames have been utilized with both of them being made of steel but the second one lacking the pin and rigid connections. These two frames have been modeled and subjected to nonlinear dynamic and loading history analysis by the assistance of ABAQUS finite element software. Amongst the output results of the analysis, the distribution of plastic hinge in the members, the rotation of plastic hinge, energy depreciation capacity can be pointed out. Using this type of modeling and conclusion, it can be discerned that the steel beam frames with beam-to-column welded rigid connections have the lowest capacity of energy depreciation, frame period, plastic hinge, rotation and early and vast formation of plastic hinge in the limbs and connections. One of the results found herein is that the general mechanism of the frame should be concluded in the investigation of the knee brace's connection in various angles with different width-to-height ratios and investigation of the hysteretic behavior's diagram.
\end{abstract}

Keywords: Nonlinear dynamic analysis, Moment frame, Finite element, Steel beam, Hysteresis diagram

\section{Introduction:}

In recent years, the use of a combined steel beam and column (CFT) system has had a considerable growth due to the exploitation of the concomitant properties of the steel's resistance and ductility in concrete's tension and hardness subject to pressure as well as application of the steel beams in the wide spans due to the high bending capacity of these beams.

Although the research on the performance of the mixed columns has been conducted since the first experiments on steel cross-sections enclosed in concrete by Burr in 1908, little progress had been made until the second half of the $20^{\text {th }}$ century. Until before 1967 , the performance of the Combined columns had been studied pivoting about an axis including the increase in the strength as a result of being enclosed and application of a tangent module solution by numerous researchers. However, the majority of the studies had been limited to circular cross-sections and no exploration had been done on the behavior of combined columns with mixed loading and different geometrical and strength specifications [1].

\section{Method of Modeling the Frames:}

In the present study, a combined knee-braced ductile moment frame with shear-yielding diameter is being investigated for the applications contingent in the earthquake-resistant designs. In this chapter, the behavior of the frame with knee bracing has been studied subject to cyclic loading. The force-displacement curve of a kneebraced frame is amongst the most important specifications in analyzing and designing steel frames. In the present study, based on the results of analysis performed according to finite element method using ABAQUS, the force-displacement curve was examined for the following parameters: the ratio of the combined column's width to thickness, knee joint's angle, brace's eccentricity and concrete's strength in the steel frame with a combined column and knee brace.

A single-storey and single-span model with a knee-braced frame as offered by Balendra et al was taken into consideration for a height of $2800 \mathrm{~mm}$ and a center-to-center span equal to $3200 \mathrm{~mm}$ and a brace length of $3726 \mathrm{~mm}$ and a cross-section of $\mathrm{H} 125 \times 125 \times 23.8(\mathrm{~kg} / \mathrm{m})$ as the orthogonal limb (column) and a cross-section of $\mathrm{H} 100 \times 100 \times 17.2(\mathrm{~kg} / \mathrm{m})$ as the horizontal limb (beam) in the frame. The specifications of the knee limb have been given in Table (2-1). The border members have been made of ASTM-A572 steel and they have been taken into account in weld modeling as the constraint "tie". 
Table 1: specifications of the element's components [2]

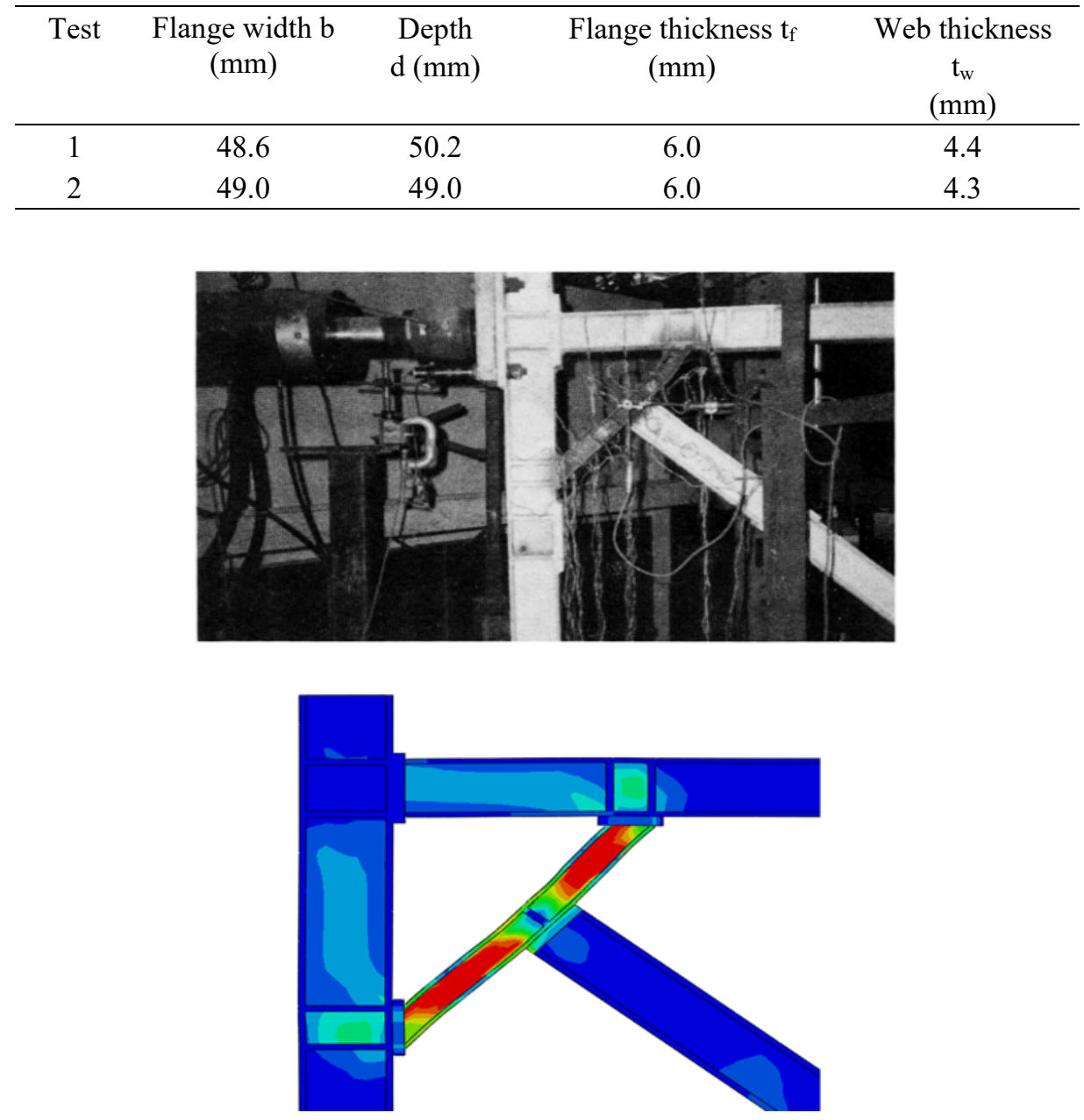

Figure (1): laboratory model [2]
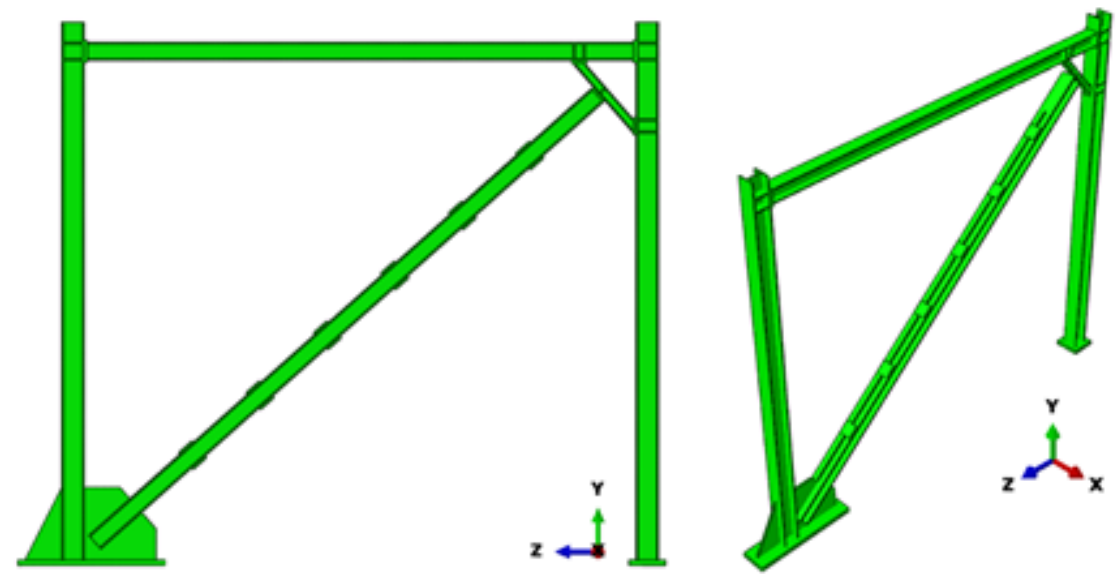

Figure (2): model constructed in ABAQUS Software [3] 


\section{Evaluation of the Analyses:}

\subsection{Stress-Strain Diagram:}

It is pertinent to the stress-strain diagram for the assumed concrete in the present study's modeling.

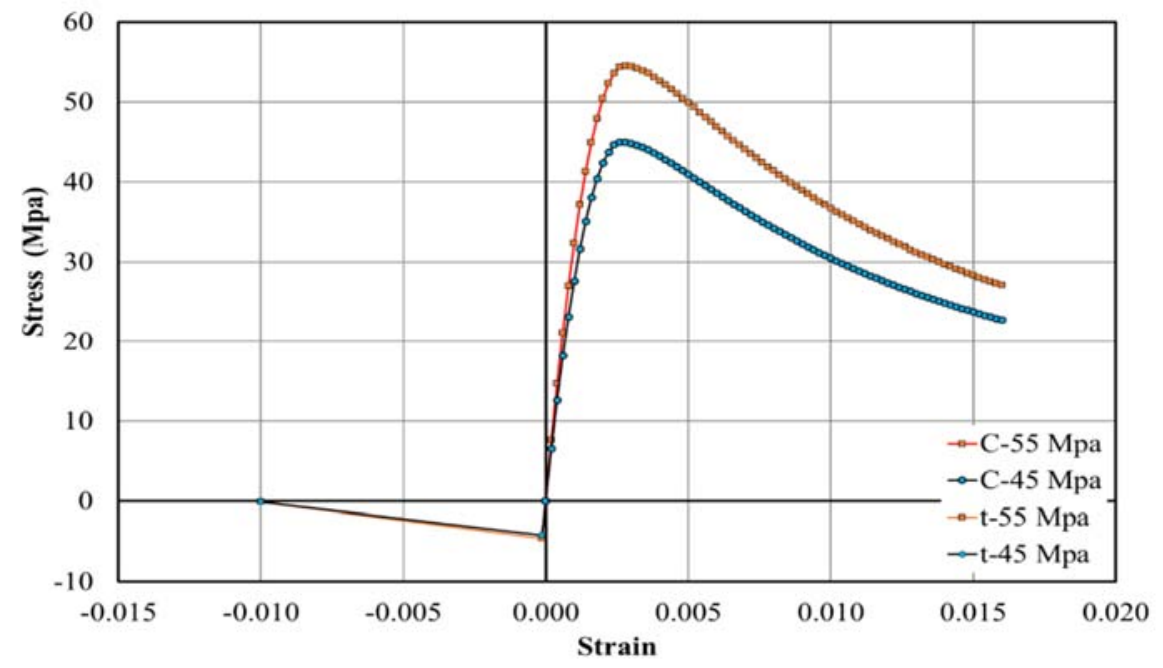

Figure (3): presumed stress-strain diagram for the models' concrete [2]

\subsection{Loading History:}

The loading history was exerted according to the pattern posited by Balendra et al that begins with consecutive cycles for measuring the variable "the column's relative displacement" and subsequently continued in the preliminary cycles with a maximum $1 \%$ increase in the relative displacement angle of the column and the exertion of loading cycle for every increase in the column's relative displacement by $1 \%$ as shown in Figure (4). The loading locus is at the tip of the column. The specimens have been subjected to cyclic loading in a semi-static manner and in displacement control mode with a low rate as specified by the software

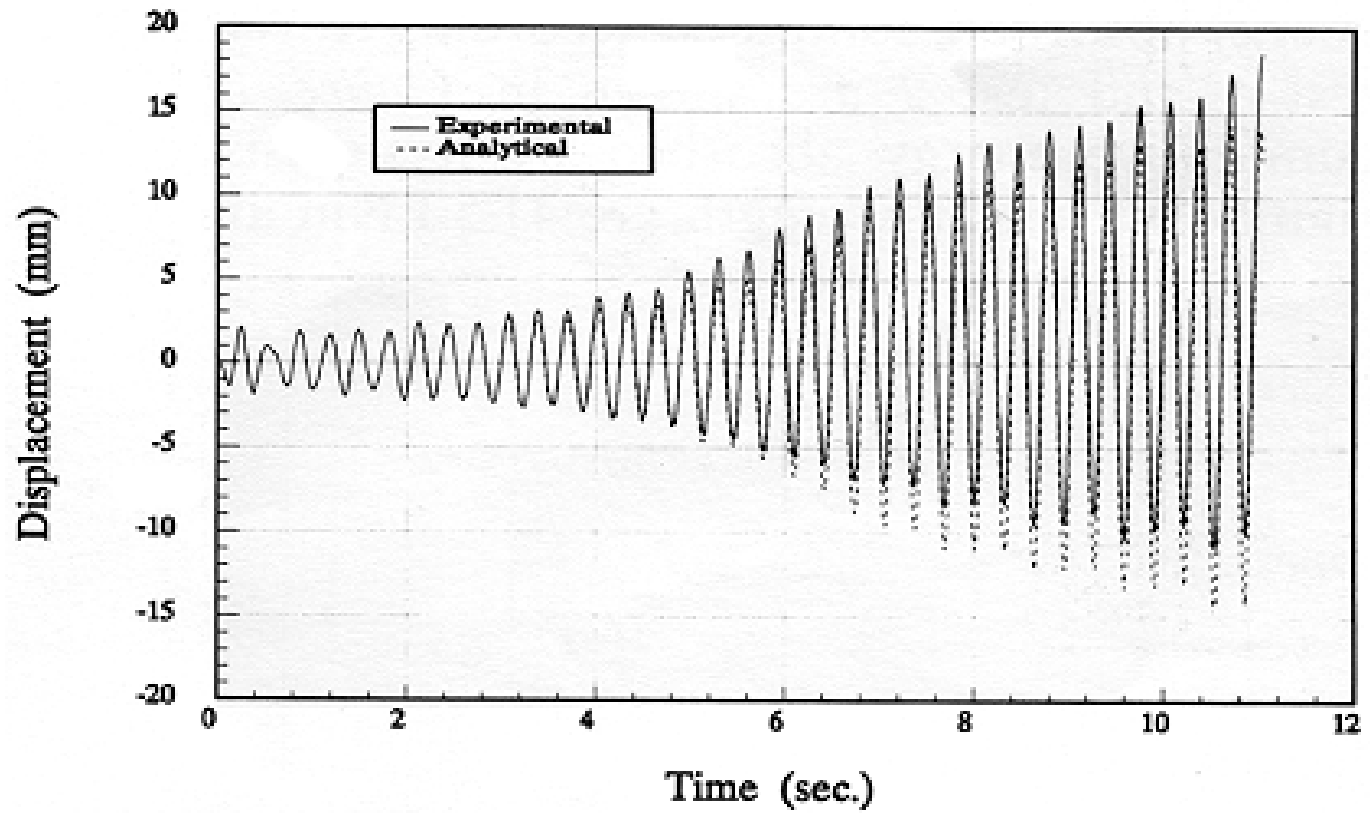

Figure (4): the diagram of the loading history according to the pattern by Balendra et al [2] 
The conditions of the modeled specimens and also their limit conditions have been considered in such a way that they can undergo deformations subject to loading similar to the deformations of the limbs in a moment frame subject to lateral loading. Based thereon, the shape of the real frame specimen tested by Balendra et al was selected and, according to the laboratory conditions, the ending sections of the beams were joined using braced plates. The columns prevent them from displacement in a stretch perpendicular to the plate.

At the foot of the columns, as well, the connections are considered in a braced form. Figure (5) displays the overall schematic configuration of the models. As it is shown in Figure (5), the boundary conditions of the loads imposed on the structure in the laboratory have been similarly used in the simulations.

The following figure shows the models' loading under boundary conditions in the software environment.
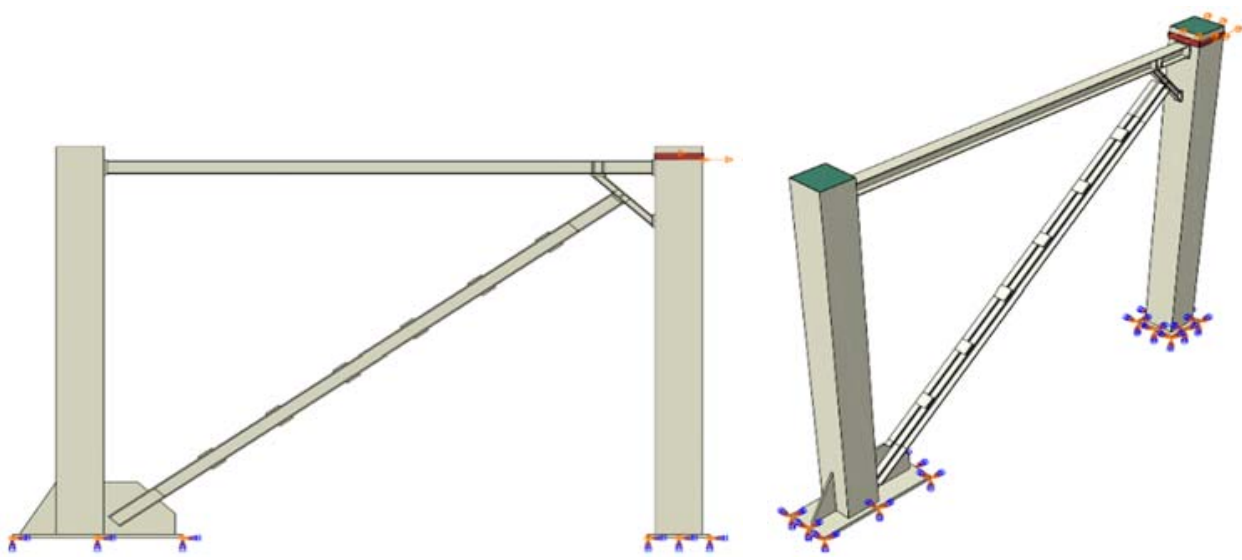

Figure (5): an example of boundary conditions and the model's loading method

In combined steel frames with joint connections, the majority of the plastic hinges are formed in lieu of in the beams in the web of the beam-to-column welds due to the concentration of tension therein indicating the brittle disintegration of such types of frames. This is while the negative buckling in the rigid frames in the region of the concrete's connection and existence of a large deal of rigidity stemming from the acceleration of concrete and weld connection causes the abrasion of the concrete and exertion of abrupt stresses to the welds in the upper side of the beam as a result of which the welds undergo disintegration and their complete energy depreciation capacity and other members' larger elastic rotations cannot be exploited in these types of frames. But, the sure thing is the higher final disintegration-resistance capacity of the combined hinged frames in contrast to the combined rigid frames. The formation of the plastic hinge in the steel beam is reflective of the intermediate ductility of such types of frames because, according to the Standard 8 of Euro Code, the locus of plastic hinge formation is in the combined concrete-beam of the combined intermediately ductile structures but the plastic hinge is formed in the steel segment of the combined beam in the highly ductile structures [4].

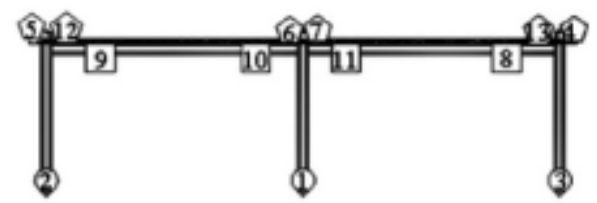

Composite Frame with Rigid Connections

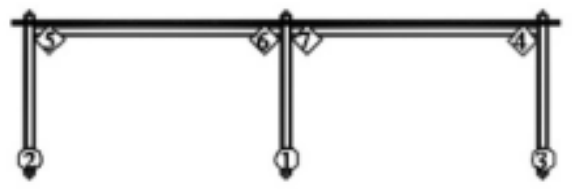

Composite Frame with Hinged Connections

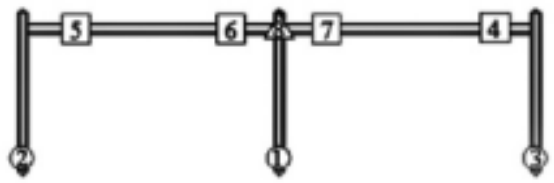

Composite Frame with Rigid Connections



Composite Frame with Hinged Connections

Figure (6): distribution of plastic hinge in the frames [5]

\section{Analyzing the Cyclic Behavior of the Models:}

In this section, the force-relative storey displacement behavioral curve and the contour of the stresses' situation are evaluated and analyzed for the specimens modeled in finite element ABAQUS Software. All of the specimens have been loaded to the storey's relative displacement that leads to the formation of joint in the knee 
and the hysteretic behavior curve that is expressive of the energy absorption of the modeled knee-braced frame specimens has been subsequently obtained. Next, the cyclic behavior of the models has been dealt with.

Cyclic Behavior of Model 1: in this section, the cyclic behavior of the knee-braced frame with a combined column has been investigated through taking various parameters into consideration as explained below. Figure (7) exhibits the force-displacement behavior of the knee-braced frame Model F-Knee-01 which, as it is observed, features an increasingly higher strength. In this frame, the knee element has been considered in a 30degree angle and a 3-millimeter thickness of the web and wing of the column as well as a 241-millimeter width of the square column.
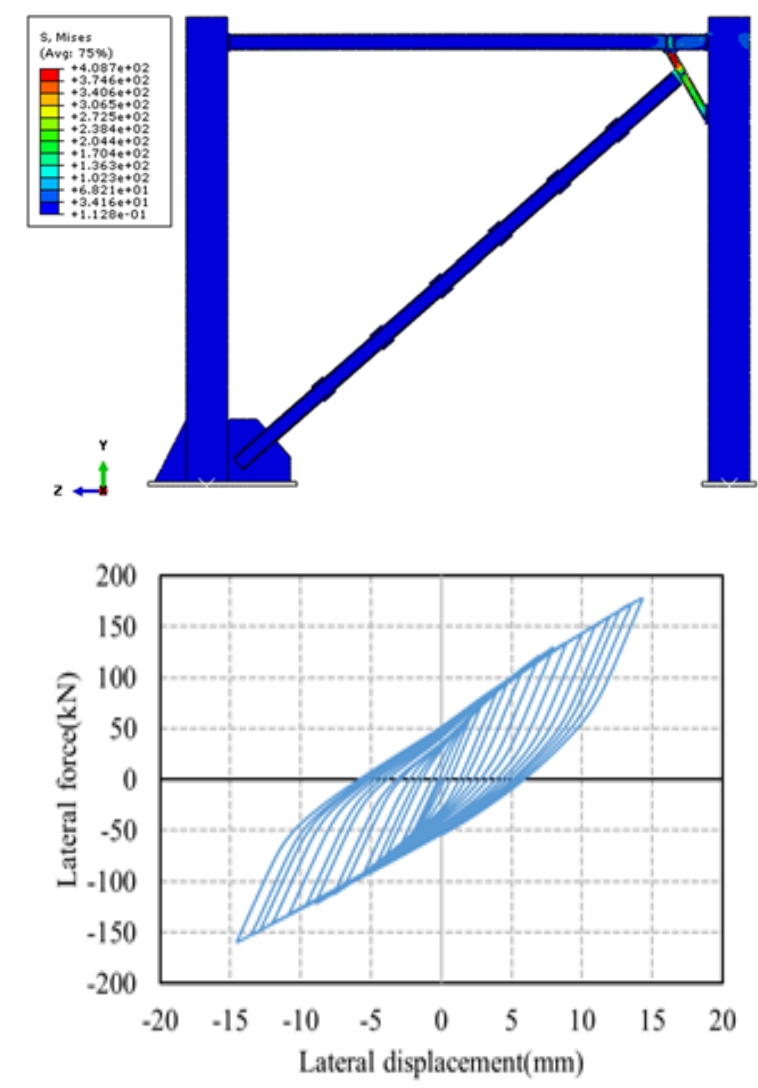

Figure (7): responses of the finite elements for F-Knee-01; A) hysteresis diagram and B) Von Mises stress in the last cycle
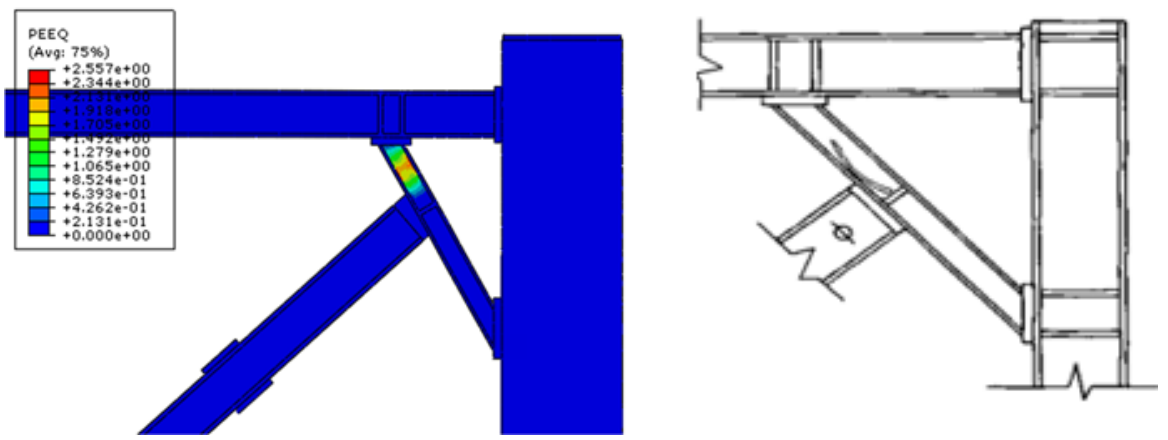

Figure (8): the disintegration mode of the F-Knee-01 specimen's web; A) laboratory state; and B) finite elements' responses 

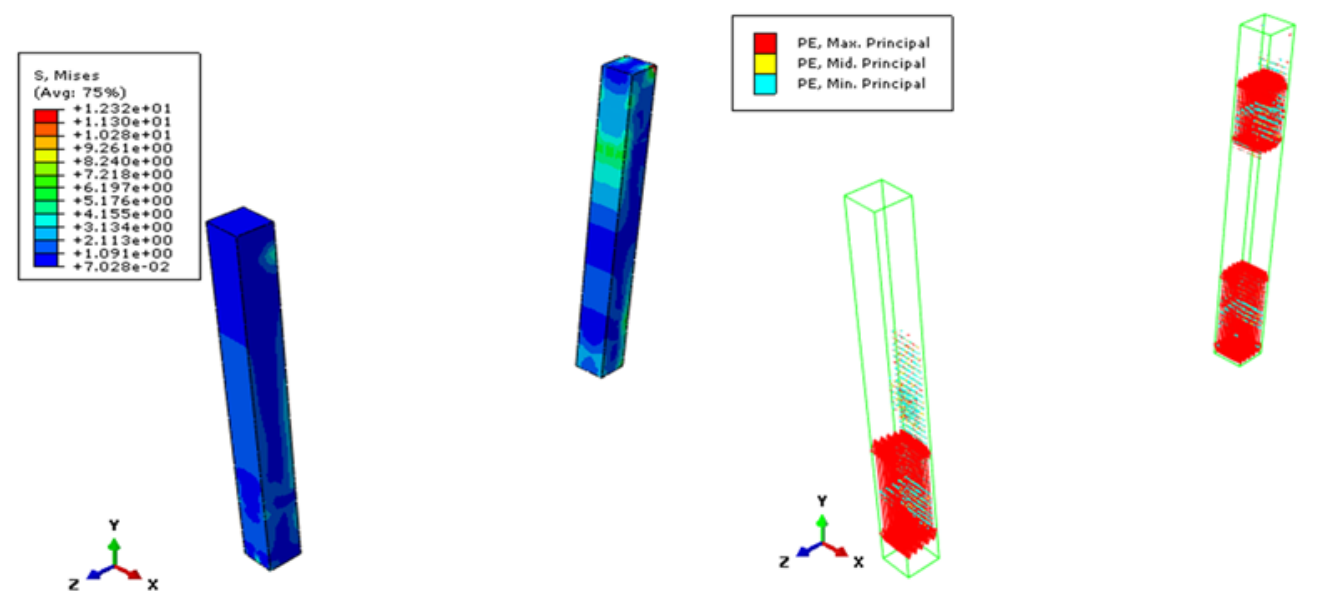

Figure (9): F-Knee-01 specimen's finite elements' responses for the column's concrete core; A) distribution of plastic strain; and B) Von Mises stress distribution

Cyclic Behavior of Model 2: in Figure (10a), the force-displacement behavior curve of the knee-braced frame, model F-Knee-02, can be seen and, as it is observed, it features an increasingly higher strength. In this frame, the knee element has been considered in a 30-degree angle and a 4-millimeter thickness of the column's web and wing. In this specimen, the column's width is $183 \mathrm{~mm}$ making it have a large cross-section in contrast to the thickness of the steel for such a reason as the change in the width of the fixed column parallel to the support of the volume of steel used.


Figure (10): F-knee-02 specimen's finite element response; A) Von Mises stress; B) equivalent plastic strain

Cyclic Behavior of Model 3: in Figure (11), another different state of the investigation can be seen. The hysteretic behavior of the knee-braced combined frame, Model F-Knee-03, has been shown in the diagram and, as it is observed, it features an increasingly higher strength. In this frame, the knee element has been considered in a 41-degree angle and a 5-millimeter thickness of the column's web and wing. In this specimen, the column's width is $148 \mathrm{~mm}$ which is increased by 0.6 in comparison to the previous column.

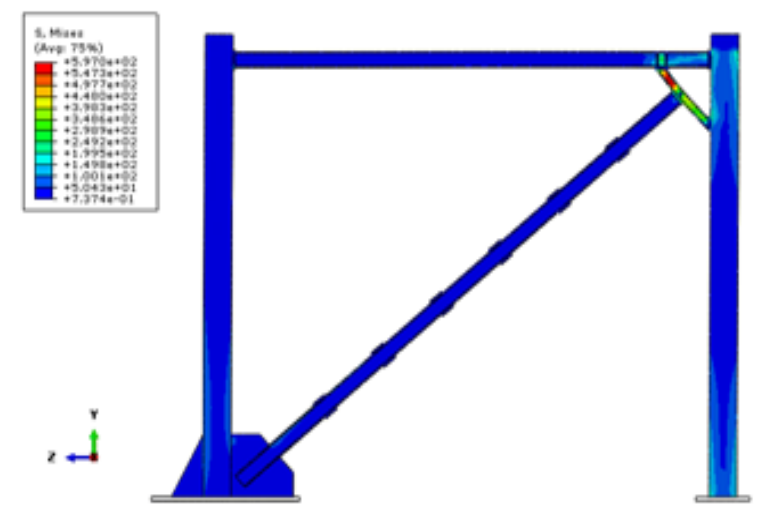




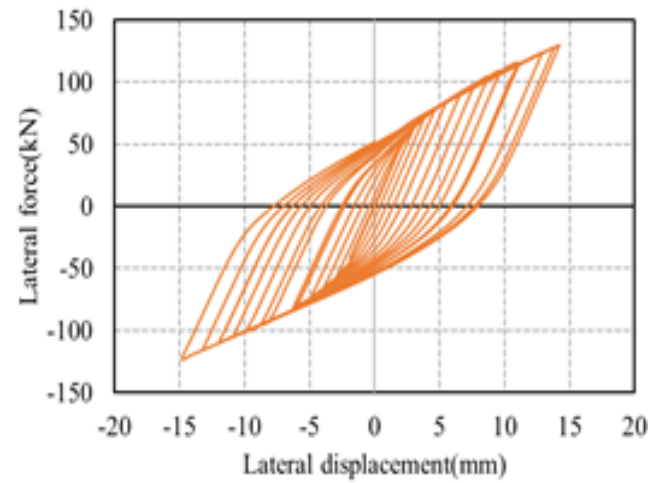

Figure (11): the finite elements' responses for F-Knee-03 Specimen; A) results obtained from hysteresis diagram and B) Von Mises stress in the combined frame

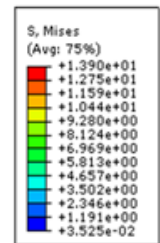<smiles>[3H][13CH]([3H])[3H]</smiles>


Figure (12): the finite elements' responses for F-Knee-03 Specimen; A) tensile damage in the concrete core and B) Von Mises stress

5. Results Obtained from the Comparison of Hysteresis Diagram:



Figure (13): comparison of hysteresis diagrams for various thicknesses and 30-degree angle 


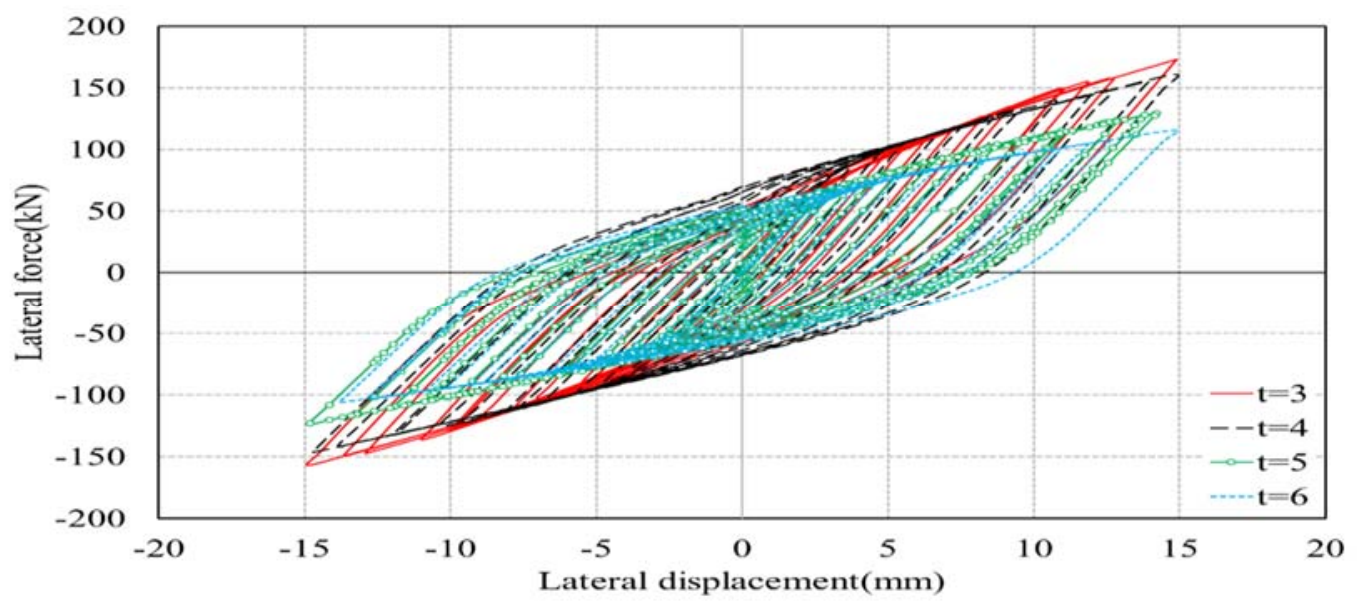

Figure (14): comparison of hysteresis diagrams for various thicknesses and 41-degree angle

\section{Conclusion:}

It can be concluded from the present study that the concrete acceleration in the periphery of the steel column and the extra hardness granted to this region has negative effect on the combined steel structures with rigid welded connections that causes the acceleration in the formation of plastic hinge in the steel beam and brittle and sudden disintegration, especially in the region welded to the column that finally bars the achievement of higher energy depreciation capacities and larger elastic rotations in other members of column and beam. Furthermore, the rigidification of the column-to-beam connection and the addition of the knee brace brings about reduction in the acceleration in the formation of plastic hinge in the structure, reduction in the plastic rotation in the connection spot, reduction in the frames' lateral displacement and reduction in the energy depreciation of the frame components and increase in the energy depreciation in the column's foot. Damage to the two regions of the knee brace is higher in 30-degree angle due to the fact that the ratio of the span to the numerical height is between these two numbers, i.e. 41 degrees, in which case, the force and stress transfer happens with more conjunction. For the steel column with a thickness of $4 \mathrm{~mm}$, the hysteresis behavior is almost identical for 41degree angle and lower for the 30-degree angle and it undergoes local narrowing as a result of the knee's buckling in 30-degree angle earlier than the other two angles. In addition, the hysteretic behavior is almost identical for 30-degree and 41-degree angles and the diagram gains a vaster area for 41-degree angle but the plastic hinge forms in the knee due to the geometrical dimensions of the frame and non-identicalness of the knee's angle and the ratio of the frame's length to width.

\section{References:}

[1] Kitada, T., (1998). "Ultimate Strength and Ductility of State-of-the-art Concrete-filled steel Bridge Piers in Japan." Engineering Structures 20(4-6): 347-354.

[2] Balendra T,Lim E L and L and Liaw C.Y(1994),"Ductile knee braced frames with Shear yielding knee for seismic resistant structures",Engineering Structures ,16(4),263-269.

[3] ABAQUS/standard user's manual,Version 9.7.2 Providence (RI): Hibbitt, Karlsson, \& Sorensen, Inc.; 2009.1

[4] Eurocode 8 (prEN 1998-1:200X). Design of structures for earthquakeresistance Part 1: General rules, seismic actions and rules for buildings2003. January 2003.

[5] G. Vasdravellis, M. Valente, C.A. Castiglioni. Behavior of exterior partial-strength composite beam-to- connections: Experimental study and numerical simulations column. Department of Structural Engineering, Politecnico di Milano, P.zza Leonardo Da Vinci 32, Italy. January 2008 